\title{
Neuronal and Glial Growth in Organotypic Cultures after Vitrification
}

\section{Amir Arav ${ }^{1 *}$, Abraham Shahar ${ }^{2}$, Ofra Ziv-Polat ${ }^{2}$, Yehudit Natan ${ }^{1}$ and Pasquale Patrizio ${ }^{3}$}

${ }^{1}$ FertileSafe, Ltd, 11 Haharash Street Ness Ziona, Israel

${ }^{2}$ NVR 11 Haharash Street, Ness Ziona, Israel

${ }^{3}$ Yale University Fertility Center, New Haven, CT, USA

\begin{abstract}
Fetal rat dorsal root ganglia (DRG) and spinal cords (SC) slices from rat fetuses were vitrified in a new semiautomatic vitrification system, cooled in sterile slush liquid air (SLA) and stored in a special sterile sealed container in liquid nitrogen (LN). Upon warming, organotypic stationary cultures were performed using NVR-Gel (composed mainly from hyaluronic acid and laminin) and enriched with neuronal factors conjugated to iron oxide nanoparticles. Evaluation of cultures was made by daily phase-contrast microscopy observations and by immunofluorescence staining.

Results revealed that SC neurons maintained their multipolar shape and regrew dendrites and axons. The round shape DRG neurons exhibited euchromatic nuclei with prominent nucleoli and an active regeneration of nerve processes. Migration of both neurons and flat cells (fibroblasts and glia Schwann cells) started within 48 hours after seeding and intensified in the upcoming days.

In conclusion, it can be said that using a semi-automatic vitrification, sterile vitrification and sterile storage of neuronal tissues from the CNS and the PNS is a successful advanced technology for the preservation of neurons and glial cells, as shown in the regain of a full regular growth pattern in culture. This may be an important step towards clinical use in the reconstruction of severe peripheral nerves and spinal cord injuries.
\end{abstract}

Keywords: Cryopreservation; Vitrification; Neurons; Spinal cord; Contamination; Regeneration

Abbreviations: BSA: Bovine serum albumin; CNS: Central Nervous System; DMEM-F12: Dulbecco's modified eagle medium-nutrient mixture F-12; DRG: Dorsal Root Ganglion; DMSO: Dimethyl Sulfoxide; EG: Ethylen Glycol; EM: Electron Microscope; ES: Equilibration Solution; FCS: Fetal Calf Serum; GDNF: Glial Cell Derived Neurotrophic Factor; HA: Hyaluronic Acid; LN: Liquid Nitrogen; NF: Neurofilament Antibodies; SLA: Slush Liquid Air; PBS: Phosphate Buffered Saline; PNS: Peripheral Nervous System; SC: Spinal Cord; SLA: Slush Liquid Air; VS: Vitrification Solution

\section{Introduction}

Vitrification is an ice free cryopreservation method which includes a step wise exposure of the sample to high concentrations of cryoprotectants (i.e. DMSO and Ethylene glycol) followed by rapid cooling to the temperature of liquid nitrogen [1-4]. As a result, the human factor is one of the most important variables affecting the success of cryopreservation procedures by vitrification $[4,5]$. Furthermore, cryopreservation of stem cells and progeny tissue by direct exposure into liquid nitrogen (LN) and their storage in standard LN tanks encompass in it the risk of potential contamination and crosscontamination by viruses, bacteria, fungi and spores that survive in LN and pose a real threat [6-8].

Therefore, the use of safe cryopreservation protocols is important and highly needed for preventing contamination of biological samples by cryogenic medium and safeguard future stem cell therapies $[9,10]$.

In order to simplify the vitrification process and to overcome these potential risks of contamination we developed three devices; (1) A semi-automatic system that allows a step wise exposure to vitrification solutions and cooling within the same device (Sarah", FertileSafe, Nes-Ziona, Israel). (2) A device for producing clean liquid air having the same temperature and properties as LN (CLAir ${ }^{\circ}$, FertileSafe, NesZiona, Israel). (3) A sterile storage device which enables storing samples in a sterile manner within the standard LN tanks, thus preserving the LN temperature and insulating the samples from being in contact with the surroundings (Esther ${ }^{\oplus}$, FertileSafe, Nes-Ziona, Israel).

\section{Materials and Methods}

\section{Preparation of organotypic DRG and SC cultures}

All the experiments were carried out and authorized by the local ethics committee for animal experimentation. Stationary organotypic DRG and spinal cord SC cultures were prepared from rat fetuses (gestational day 15, Lewis inbred, Harlan, Israel). Immediately after dissection, the isolated DRG and SC slices were cut with a McIlwain tissue chopper (Ted-Pella, California, USA) into small slices (of 400 $\mu \mathrm{m}$ thickness). At a desired time, tissue were warmed and seeded in 12 well culture plates containing $1 \mathrm{~mL}$ of NVR-Gel (NVR laboratories, Nes-Ziona, Israel). Cultures were enriched with glial cell derived neurotrophic factor (GDNF) conjugated iron oxide nanoparticles (10 $\mu \mathrm{g} / \mathrm{mL}$ final concentration). Once embedded and seeded in the gel, cultures were subsequently fed with the nutrient medium every 4 days. Monitoring of the DRG-SC growth pattern was done by daily phase contrast microscopic observations starting 24 hours after setting the cultures onward. Immunofluorescence staining of cultures was made at different stages of cultivation.

*Corresponding author: Amir Arav, Fertile Safe, Ltd, 11 Haharash street Ness Ziona Israel, Tel: 3568103636; E-mail: dity2@netvision.net.il

Received August 25, 2015; Accepted September 30, 2015; Published October 02, 2015

Citation: Arav A, Shahar A, Ziv-Polat O, Natan Y, Patrizio P (2015) Neuronal and Glial Growth in Organotypic Cultures after Vitrification. J Stem Cell Res Ther 5: 308. doi:10.4172/2157-7633.1000308

Copyright: (c) 2015 Arav A, et al. This is an open-access article distributed under the terms of the Creative Commons Attribution License, which permits unrestricted use, distribution, and reproduction in any medium, provided the original author and source are credited. 


\section{Culture medium}

The culture medium was prepared from $90 \%$ Dulbecco's modified eagle medium-nutrient mixture F-12 (DMEM-F12), 10\% heatdenatured fetal calf serum (FCS), $6 \mathrm{~g} / \mathrm{L}$ D-glucose, $2 \mathrm{nM}$ glutamine, 25 $\mu \mathrm{g} / \mathrm{mL}$ gentamycin, and $50 \mathrm{ng} / \mathrm{mL}$ IGF-I (all purchased from Biological Industries, Bet-Haemek, Israel).

\section{NVR-Gel for neuronal cultivation}

NVR-Gel (NVR Laboratories, Nes-Ziona, Israel) is a proprietary gel composed of two main components: high molecular hyaluronic acid (HA, $3 \times 10^{6} \mathrm{Da}$ ) and laminin, both known to be inherent elements of the extracellular matrix. For cell cultivation, HA of 1\% (Bio-Technology General, Kiryat Malachi, Israel) was diluted with culture medium to the concentrations of $0.3-0.5 \%$. Laminin (Sigma, St. Louis, Missouri, USA) was then mixed with the diluted HA (final concentration of $10 \mu \mathrm{g} / \mathrm{mL}$ ). GDNF, free or conjugated to iron oxide nanoparticles, was finally added (10 ng/mL), to complete the NVR-Gel composition.

\section{Immunofluorescence staining}

Samples were fixed in $4 \%$ paraformaldehyde. The fixed cells were permeabilized with $0.1 \%$ of Triton X-100 in PBS and then immunoblocked with a $1 \%$ bovine serum albumin (BSA) in PBS for 1 hour at room temperature. The specimens were then double stained with mouse anti S-100 antibodies (1:80, Acris Antibodies, glial cell marker) and rabbit anti neurofilament antibodies (NF, Novus Biologicals, 1: 500, neuronal cell marker). The primary antibodies were diluted in $0.1 \%$ BSA and $0.05 \%$ Tween-20 in PBS (diluents buffer) and incubated with the specimens overnight at $4^{\circ} \mathrm{C}$. After rinsing with $0.05 \%$ Tween-20 in PBS (wash buffer), the DRG specimens were incubated for $1 \mathrm{~h}$ at room temperature with the appropriate fluorescently labeled secondary antibodies.

\section{Vitrification, storage and warming methods}

A new device for semi-automatic vitrification was used (Sarah, Fertile Safe, Ness Ziona, Israel). The device is equipped with a special capsule containing electron microscope (EM) gold grid connected to $0.25 \mathrm{ml}$ IMV straw (Figure 1) (IMV Technologies, L'Aigle, France). Six straws, each containing 6 tissue slices, were placed in a special mixing chamber (Figure 2). An increasing concentration of vitrification solution was continuously infused using a syringe pump (New Era Pump Systems, NY, USA) for 25 minutes with equilibration solution (ES) (Sage ${ }^{\mathbb{B}}$ Vitrfication kit, Origio, Malov, Denmark). Then vitrification solution (VS) (Sage ${ }^{\oplus}$ Vitrfication kit, Origio, Malov, Denmark) was flowed through the samples for 5 minutes. Finally samples were plunged into SLA $\left(-208^{\circ} \mathrm{C}\right)$ (Clair ${ }^{\circ}$ and VitMaster ${ }^{\ominus}$, FertileSafe, Nes-Ziona, Israel). The samples were stored in a special sterile sealed container (Esther ${ }^{\oplus}$, FertileSafe, Nes-Ziona, Israel) for 1week. For the warming, straws were held for 10 second in air before being plunged into $80^{\circ} \mathrm{C}$ warming solution (1M sucrose) for 1 second and immediately placed in $37^{\circ} \mathrm{C}$ warming solution ( $1 \mathrm{M}$ sucrose) for 2.5 minutes. Then samples were moved into decreased sucrose concentrations $(0.5 \mathrm{M} \& 0.25 \mathrm{M}$ for 2.5 minutes in each solution) (Sage $e^{\bullet}$ vitrification warming kit, Origio, Malov, Denmark).

\section{Results}

The new semi-automatic vitrification device allows gradual increase of the vitrification solution which permits both standardization of the vitrification process and reduction of the tissue damages generally seen during the vitrification procedure. This device enables to vitrify up to 6 straws and 36 slices (6x6 tissue

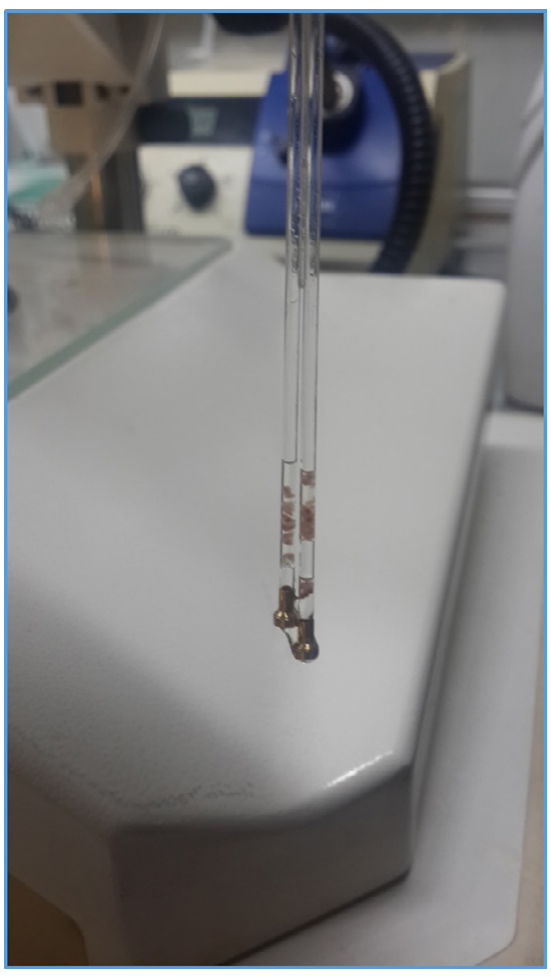

Figure 1: Spinal cord tissue (6 slices/straw) inside two straws with gold coated mesh capsule (Sarah $®$, FertileSafe, Israel).

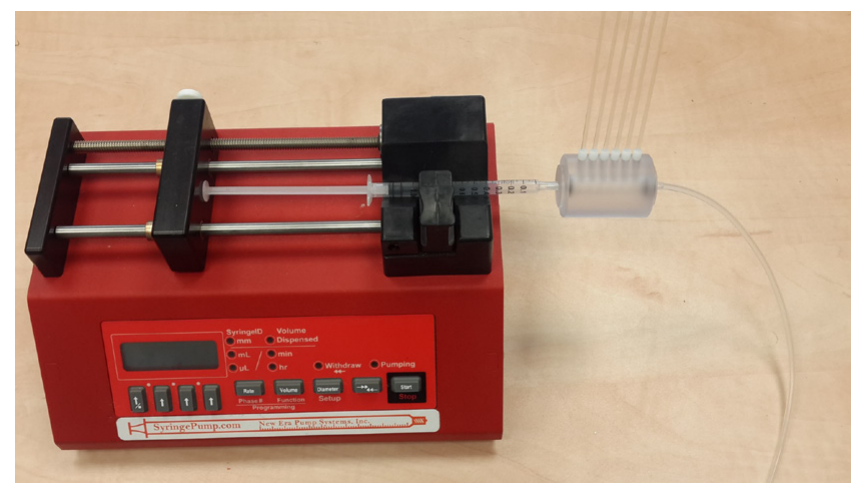

Figure 2: Straws $(n=6)$ are placed in a vitrification solution mixing chamber and are loaded gradually with increasing concentrations of vitrification solution.

slices) simultaneously, thus increasing the efficiency of the process. The exposure of neuronal tissue slices to the sterile SLA $\left(-208^{\circ} \mathrm{C}\right)$ enabled vitrification and did not damage the neurons and glia cells as seen by post warming growth. Furthermore, both neuronal cell types maintained their multipolar and round shapes (Figure 3B,C) and exhibited intensive fiber regeneration. DRG neurons exhibited one or two euchromatic nuclei with prominent nucleoli (Figure 3C). Several nerve cells showed regeneration of short nerve processes and a thin long process which might be probably the axon (Figure $3 \mathrm{~A})$. The flat glia and fibroblast cells migrated from the explants and divided actively (Figure 4A,B). Migration from explants of both neurons and flat cells (fibroblasts and glia Schwann cells) started within 48 hours after seeding and intensified in the upcoming 


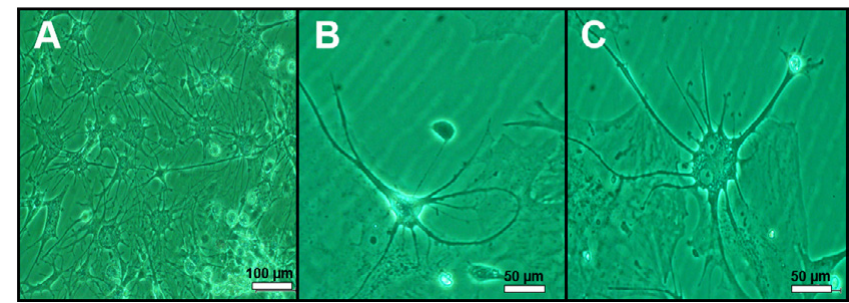

Figure 3: Phase-contrast microscopy of SC and DRG slices cultured in NVR-Ge 8 days after vitrification. (A) Neuronal and glia migration (B) A round DRG neuron which exhibits regenerated nerve fibers, euchromatic nucleus with a prominent nucleolus. (C) A multipolar SC nerve cell with regenerated nerve fibers.

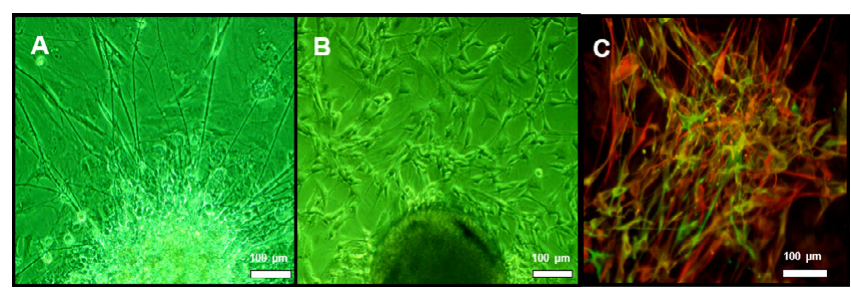

Figure 4: Regeneration of nerve fibers and migration of neuronal and glial cells from SC and DRG slices of rat fetuses cultured for 3 days (A\&B) and 15 days (C) in NVR gel after vitrification. (A\&B) Phase-contrast microscopy, (C) immunefluorescent double staining of migrated cells. Glial cells are marked in red (anti S-100 antibodies) and neurons in green (anti neurofilament antibodies).

days, and anti neurofilament antibodies (NF, neuronal cell marker, (Figure 4C).

\section{Discussion}

Vitrification has been shown to be superior to slow freezing for the success in cryopreservation of various tissues [11-14]. However, manual operation and the risk of contamination posed by liquid nitrogen limits the practical application and preservation of stem cells and tissues. It is likely that safe and efficient cryopreservation of neural cells will be a prerequisite for quality assurance of the cells for clinical use [3]. This is the first report on a semi-automatic and a sterile vitrification procedure applied to neuronal spinal cord tissue of the central nervous system and the dorsal root ganglia of the peripheral nervous system. The semi-automatic device allows a gradual exposure of the tissues to an increasing concentration of vitrification solution without the need to move the tissues manually between the equilibration and vitrification solutions.

In conclusion we can say that using a semi-automatic vitrification device followed by plunging into sterile SLA and storing in sterile canister results in the full regeneration and growth in culture of neuronal and glia cells from fetuses (SC) and (DRG) explants and can play an important role in clinical use for the reconstruction of severe peripheral nerves and spinal cord injurie [15].

\section{Competing Interests}

Arav Amir and Patrizio Pasquale are founders of FertileSafe, Natan Yehudit is an employee of FertileSafe. Shahar Abraham and Ziv-Polat Ofra are employees of NVR.

\section{Authors' Contributions}

Arav Amir and Ziv-Polat Ofra carried out the experiments, ZivPolat Ofra synthesized the nanoparticles and conjugate them to neuronal factors. Abraham Shahar dissected the fetal SCs and DRGs and analyzed the interpretation of the data with Arav Amir. Natan Yehudit, Arav Amir and Patrizio Pasquale wrote the manuscript.

\section{References}

1. Fahy GM, Saur J, Williams RJ (1990) Physical problems with the vitrification of large biological systems. Cryobiology 27: 492-510. [PubMed]

2. Arav A (1992) Vitrification of oocyte and embryos, In Lauria, A, Gandolfi, F. (eds) " New trends in embryo transfer" Portland press, Cambridge England 255-264.

3. Arav A, Yavin S, Zeron Y, Natan D, Dekel I, et al. (2002) New trends in gamete's cryopreservation. Mol Cell Endocrinol 187: 77-81. [PubMed]

4. Kuleshova L, Tan F, Magalha S, Gouk S, Lee KH, Dawe GS (2009) Effective Cryopreservation of Neural Stem or Progenitor Cells Without Serum or Proteins by Vitrification. Cell Transplantation 18: 135-144.

5. Saragusty J, Arav A (2011) Current progress in oocyte and embryo cryopreservation by slow freezing and vitrification. Reproduction 141: 1-19. [PubMed]

6. Arav A, Natan $Y$ (2013) Vitrification of oocytes: from basic science to clinical application. Adv Exp Med Biol 761: 69-83. [PubMed]

7. Bielanski A, Vajta G (2009) Risk of contamination of germplasm during cryopreservation and cryobanking in IVF units. Hum Reprod 24: 2457-2467. [PubMed]

8. Grout BW, Morris GJ (2009) Contaminated liquid nitrogen vapour as a risk factor in pathogen transfer. Theriogenology 71: 1079-1082. [PubMed]

9. Bielanski A (2012) A review of the risk of contamination of semen and embryos during cryopreservation and measures to limit cross-contamination during banking to prevent disease transmission in ET practices. Theriogenology 77 : 467-82.

10. Kurita N, Frassoni F, Chiba S, Podestà M (2015) Impact of length of cryopreservation and origin of cord blood units on hematologic recovery following cord blood transplantation. Bone Marrow Transplant 50: 818-821. [PubMed]

11. Tournaye H, Dohle GR, Barratt CL (2014) Fertility preservation in men with cancer. Lancet 384: 1295-1301. [PubMed]

12. Song YC, Hagen PO, Lightfoot FG, Taylor MJ, Smith AC, et al. (2000) In vivo evaluation of the effects of a new ice-free cryopreservation process on autologous vascular grafts. J Invest Surg 13: 279-288. [PubMed]

13. Tan FC, Lee KH, Gouk SS, Magalhaes R, Poonepalli A, et al. (2007) Optimization of cryopreservation of stem cells cultured as neurospheres: comparison between vitrification, slow-cooling and rapid cooling freezing protocols. Cryo Letters 28: 445-460. [PubMed]

14. Herraiz S, Novella-Maestre E, Rodríguez B, Díaz C, Sánchez-Serrano M Mirabet V, et al. (2014) Improving ovarian tissue cryopreservation for oncologic patients: slow freezing versus vitrification, effect of different procedures and devices. Fertil Steril 101:775-84.

15. Ziv-Polat O, Shahar A, Levy I, Skaat H, Neuman S, et al. (2014) The role of neurotrophic factors conjugated to iron oxide nanoparticles in peripheral nerve regeneration: in vitro studies. Biomed Res Int 2014: 267808. 